\title{
Characterization of Lifetime Distribution Based on Generalized Interval Entropy
}

\author{
Vikas Kumar $^{1, *}$, Nirdesh Singh ${ }^{2}$ \\ ${ }^{1}$ Department of Applied Sciences, UIET, M. D. University, India \\ ${ }^{2}$ Department of Electrical Engineering, Deenbandhu Chhotu Ram University of Science and Technology Murthal, India
}

\begin{abstract}
In this paper, we introduce the concept of generalized interval entropy measure of order $\alpha$ and type $\beta$ for the doubly truncated random variable. A characterization problem for the generalized interval entropy measure has been studied. The exponential, the pareto and finite range distributions have been characterized in terms of the proposed entropy measure and generalized failure rate function. A lower bound to generalized interval entropy has also been derived. Finally some stochastic comparison based on generalized interval entropy are presented.
\end{abstract}

Keywords Shannon entropy, Interval entropy, Generalized failure rate, Characterization.

AMS 2010 subject classifications 62E10; 62N05

DOI: $10.19139 /$ soic.v6i4.328

\section{Introduction}

In the literature, the problem of characterizing probability distributions has been investigated by many researchers, refer to Galambos and Kotz [10] and Azlarov and Volodin [3] among others. The standard practice in modeling statistical data is either to derive the appropriate model based on the physical properties of the system or to choose a flexible family of distributions and then find a member of the family that is appropriate to the data. In both situations it would be helpful if we find characterization theorems that explain the distribution using important measures of indices.

In modeling and analysis of lifetime data, the average amount of uncertainty associated with the random variable $X$, as given by Shannon entropy, is

$$
H(X)=-\int_{0}^{\infty} f(x) \log f(x) d x
$$

Since the work of Shannon [19], the measure of entropy has been generalized in a number of different ways by different researchers. A generalization of order $\alpha$ and type $\beta$ of the entropy (1) is the Verma's entropy [21] defined as

$$
H_{\alpha}^{\beta}(X)=\frac{1}{\beta-\alpha} \log \left[\int_{0}^{\infty} f^{\alpha+\beta-1}(x) d x\right] ; \beta-1<\alpha<\beta, \beta \geq 1
$$

When $\beta=1$ and $\alpha \longrightarrow 1, H_{\alpha}^{\beta}(X) \longrightarrow H(X)$ given in (1). Varma's entropy measure is much more flexible due to the parameters $\alpha$ and $\beta$, enabling several measurements of uncertainty within a given distribution and increase the

\footnotetext{
*Correspondence to: Vikas Kumar (Email: vikas_iitr82@yahoo.co.in). Department of Applied Sciences, UIET, M. D. University, Rohtak124001, India
}

ISSN 2310-5070 (online) ISSN 2311-004X (print)

Copyright (C) 2018 International Academic Press 
scope of application. The residual lifetime of the system when it is still operating at time $t$ is $X_{t}=(X-t \mid X>t)$; Ebrahimi [8] proposed the entropy of the residual lifetime $X_{t}$ as

$$
H(X ; t)=-\int_{t}^{\infty} \frac{f(x)}{\bar{F}(t)} \log \frac{f(x)}{\bar{F}(t)} d x, t>0
$$

Ebrahimi [8] showed that the dynamic measure (3) determines the underlying distribution function uniquely. Similar results in case of a generalized residual entropy have been derived by Belzunce et al. [5] and Asadi et al. [1]. Since the characterizations of distributions have numerous applications in reliability, characterizations in terms of dynamic entropies of various distributions have been proposed in the literature, refer to, Nair and Rajesh [14] and Asadi and Ebrahimi [2].

However, in many realistic situations, uncertainty is not necessarily related to the future but can also refer to the past. Based on this idea, Di Crescenzo and Longobardi [6] introduced an entropy-based measure of uncertainty in past life time $X_{t}^{*}=[t-X \mid X \leq t]$, and called it past entropy. The measures of uncertainty in context with past lifetime distributions have been studied extensively in the literature, refer to, Di Crescenzo and Longobardi [7] Nanda and Paul [15] and Kumar et al. [11].

In many situations, we only have information between two points, so we should study the statistical measures under the condition of doubly truncated random variables. The doubly truncated measures are applicable to engineering systems when the observations are measured after it starts operating and before it fails. If the random variable $X$ denotes the lifetime of a unit, then the random variable $\left(X \mid t_{1}<X<t_{2}\right)$; where $\left(t_{1}, t_{2}\right) \in D=\left\{(u, v) \in \Re^{2}: F(u)<F(v)\right\}$ is called a doubly truncated lifetime variable. Another extension of Shannon entropy is based on a doubly truncated random variable $\left(X \mid t_{1}<X<t_{2}\right)$, which is defined as

$$
H\left(X ; t_{1}, t_{2}\right)=-\int_{t_{1}}^{t_{2}} \frac{f(x)}{\bar{F}\left(t_{1}\right)-\bar{F}\left(t_{2}\right)} \log \frac{f(x)}{\bar{F}\left(t_{1}\right)-\bar{F}\left(t_{2}\right)} d x
$$

Given that a system has survived up to time $t_{1}$ and has been found to be down at time $t_{2}$, then $H\left(X ; t_{1}, t_{2}\right)$ measure the uncertainty about its lifetimes between $t_{1}$ and $t_{2}$. Sunoj et al. [20] have explored the use of information measures for double truncated random variables. Furthermore, Misagh and Yari [12, 13] explored the use of weighted information measures for doubly truncated random variables. For various results on doubly truncated random variable, we refer to Kayal and Moharana [24], and Kundu [23].

Since generalized entropy plays an important role, in the field of reliability theory and survival analysis, when a system has lifetime between two time points $\left(t_{1}, t_{2}\right)$. The measure proposed for doubly truncated random variable appears in quasar survey, where an investigator assumes that the apparent magnitude is doubly truncated. Also, the times to progression for patients with certain disease who received chemotherapy, experienced tumor progression and subsequently died, are doubly truncated. Motivated with this usefulness of the generalized entropy and the interval entropy, in the present note, an attempt is made to derive some new characterizations to certain probability distributions and families of distributions using generalized interval entropy, which are useful for modeling and analysis of lifetime data.

The paper is organized as follows. In Section 2 , we propose the generalized interval entropy and show that the effect of monotone transformations on it. Section 3 is devoted to the characterization result and uniform distribution has been characterized through the generalized interval entropy. In Section 4, we characterize the lifetime distributions based on relationship between the proposed dynamic entropy measure $H_{\alpha}^{\beta}\left(X ; t_{1}, t_{2}\right)$ and generalized failure rate function, and also we derive an lower bound to the generalized interval entropy. Some stochastic comparison based on generalized interval entropy are presented in Section 5. 


\section{Generalized Interval Entropy}

The generalized interval entropy of order $\alpha$ and type $\beta$ of the doubly truncated random variable $\left(X \mid t_{1}<X<t_{2}\right)$ is proposed as

$$
H_{\alpha}^{\beta}\left(X ; t_{1}, t_{2}\right)=\frac{1}{\beta-\alpha} \log \left[\int_{t_{1}}^{t_{2}}\left(\frac{f(x)}{F\left(t_{2}\right)-F\left(t_{1}\right)}\right)^{\alpha+\beta-1} d x\right] ; \beta-1<\alpha<\beta, \beta \geq 1 .
$$

This can be rewritten as

$$
\int_{t_{1}}^{t_{2}} f^{\alpha+\beta-1}(x) d x=\left(F\left(t_{2}\right)-F\left(t_{1}\right)\right)^{\alpha+\beta-1} \exp \left[(\beta-\alpha) H_{\alpha}^{\beta}\left(X ; t_{1}, t_{2}\right)\right] .
$$

When the system has the age $t_{1}$, for different value of $\alpha$ and $\beta$, the generalized interval entropy $H_{\alpha}^{\beta}\left(X ; t_{1}, t_{2}\right)$ provide the information spectrum of the system remaining life until age $t_{2}$.

When, $t_{2} \rightarrow \infty$, and $t_{1} \rightarrow 0$, then (5) reduce respectively to generalized residual and past entropy measure, which are given respectively as

$$
H_{\alpha}^{\beta}\left(X ; t_{1}\right)=\frac{1}{\beta-\alpha} \log \left(\frac{\int_{t_{1}}^{\infty} f^{\alpha+\beta-1}(x) d x}{\bar{F}^{\alpha+\beta-1}\left(t_{1}\right)}\right)
$$

and

$$
\bar{H}_{\alpha}^{\beta}\left(X ; t_{2}\right)=\frac{1}{\beta-\alpha} \log \left(\frac{\int_{0}^{t_{2}} f^{\alpha+\beta-1}(x) d x}{F^{\alpha+\beta-1}\left(t_{2}\right)}\right)
$$

refer to Baig and Dar [4].

Next we show that the effect of monotone transformations on generalized interval entropy defined in (5). In this context we prove the following result.

\section{Theorem 1}

Let $X$ be a non-negative and continuous random variables with p.d.f. $f$ and distribution function $F$. Let $Y=\phi(X)$, with $\phi$ be a strictly monotonic increasing, continuous and differentiable function, with derivative $\phi^{\prime}$. Then for all $0<t_{1}<t_{2}<\infty$, we have

$$
H_{\alpha}^{\beta}\left(Y ; t_{1}, t_{2}\right)=H_{\alpha}^{\beta}\left(X ; \phi^{-1}\left(t_{1}\right), \phi^{-1}\left(t_{2}\right)\right)+\left(\frac{2-\alpha-\beta}{\beta-\alpha}\right) \log \phi^{\prime}(x) .
$$

Proof The probability density function of $Y=\phi(X)$ is $g(y)=\frac{f\left(\phi^{-1}(y)\right)}{\phi^{\prime}\left(\phi^{-1}(y)\right)}$. Thus

$$
H_{\alpha}^{\beta}\left(Y ; t_{1}, t_{2}\right)=\frac{1}{\beta-\alpha} \log \left[\int_{t_{1}}^{t_{2}}\left(\frac{g(y)}{G\left(t_{2}\right)-G\left(t_{1}\right)}\right)^{\alpha+\beta-1} d y\right] ; \beta-1<\alpha<\beta, \beta \geq 1 .
$$

This gives

$$
H_{\alpha}^{\beta}\left(Y ; t_{1}, t_{2}\right)=\frac{1}{\beta-\alpha} \log \left[\int_{t_{1}}^{t_{2}}\left(\frac{f\left(\phi^{-1}(y)\right)}{F\left(\phi^{-1}\left(t_{2}\right)\right)-F\left(\phi^{-1}\left(t_{1}\right)\right)}\right)^{\alpha+\beta-1}\left(\frac{1}{\phi^{\prime}\left(\phi^{-1}(y)\right)}\right)^{\alpha+\beta-1} d y\right] .
$$

By taking $x=\phi^{-1}(y)$, we obtain

$$
H_{\alpha}^{\beta}\left(Y ; t_{1}, t_{2}\right)=\frac{1}{\beta-\alpha} \log \left[\int_{\phi^{-1}\left(t_{1}\right)}^{\phi^{-1}\left(t_{2}\right)}\left(\frac{f(x)}{F\left(\phi^{-1}\left(t_{2}\right)\right)-F\left(\phi^{-1}\left(t_{1}\right)\right)}\right)^{\alpha+\beta-1}\left(\frac{1}{\phi^{\prime}(x)}\right)^{\alpha+\beta-2} d x\right]
$$


This can be written as

$$
H_{\alpha}^{\beta}\left(Y ; t_{1}, t_{2}\right)=H_{\alpha}^{\beta}\left(X ; \phi^{-1}\left(t_{1}\right), \phi^{-1}\left(t_{2}\right)\right)+\left(\frac{2-\alpha-\beta}{\beta-\alpha}\right) \log \phi^{\prime}(x) .
$$

This prove the result.

\section{Remark 1}

For any absolutely continuous random variable $X$, define $Y=a X+b$, where $a>0$ and $0<b<t_{1}$ are constants. Then

$$
H_{\alpha}^{\beta}\left(Y ; t_{1}, t_{2}\right)=H_{\alpha}^{\beta}\left(X ; \frac{t_{1}-b}{a}, \frac{t_{2}-b}{a}\right)+\left(\frac{2-\alpha-\beta}{\beta-\alpha}\right) \log a .
$$

Thus generalized interval entropy defined in (5) is invariant under location but not under scale transformation.

\section{Example 1}

Pareto distribution plays a central role in various applications. It is used in the investigation of city population, occurrence of natural resources, insurance risk, size of human settlements, reliability modeling and business failure. It has been an important model in many socio-economic studies. Let $X$ be a random variable having classical Pareto distribution with pdf

$$
f(x)=\frac{\theta}{x^{\theta+1}}, x \geq 1, \theta>0 .
$$

Thus generalized interval entropy (5) of for classical Pareto distribution is given as

$$
H_{\alpha}^{\beta}\left(X ; t_{1}, t_{2}\right)=\frac{1}{\beta-\alpha} \log \left(\frac{\theta^{\alpha+\beta-1}}{\left(t_{1}^{-\theta}-t_{2}^{-\theta}\right)^{\alpha+\beta-1}} \int_{t_{1}}^{t_{2}} x^{-(\theta+1)(\alpha+\beta-1)} d x\right)
$$

which gives,

$$
H_{\alpha}^{\beta}\left(X ; t_{1}, t_{2}\right)=\frac{1}{\beta-\alpha} \log \left[\left(\frac{\theta^{\alpha+\beta-1}}{(\theta+1)(1-\alpha-\beta)+1}\right)\left(\frac{t_{2}^{(\theta+1)(1-\alpha-\beta)+1}-t_{1}^{(\theta+1)(1-\alpha-\beta)+1}}{\left(t_{1}^{-\theta}-t_{2}^{-\theta}\right)^{\alpha+\beta-1}}\right)\right] .
$$

\section{Example 2}

Let $X$ be a random variable follows the power distribution with cumulative distribution function (cdf)

$$
F(x)=\left(\frac{x}{a}\right)^{b}, 0<x<a, b>0 .
$$

Thus we expressed generalized interval entropy (5) for power distribution, which is given as follows

$$
H_{\alpha}^{\beta}\left(X ; t_{1}, t_{2}\right)=\frac{1}{\beta-\alpha} \log \left[\left(\frac{b^{\alpha+\beta-1}}{a^{b(\alpha+\beta-1)}[(b-1)(\alpha+\beta-1)+1]}\right)\left(\frac{t_{2}^{(b-1)(\alpha+\beta-1)+1}-t_{1}^{(b-1)(\alpha+\beta-1)+1}}{\left(\left(\frac{t_{2}}{a}\right)^{b}-\left(\frac{t_{1}}{a}\right)^{b}\right)^{\alpha+\beta-1}}\right)\right] .
$$

Example 3

If $\mathrm{X}$ is Folded Cramer Distribution with probability density function $f(x)=\frac{\theta x}{(1+\theta x)^{2}}$, and survival function $\bar{F}(x)=\frac{1}{(1+\theta x)}$, then generalized interval entropy (5) is

$$
\begin{aligned}
H_{\alpha}^{\beta}\left(X ; t_{1}, t_{2}\right) & =\frac{1}{\beta-\alpha}\left[\log \left(\frac{1}{\left(\frac{t_{2}}{1+\theta t_{2}}-\frac{t_{1}}{1+\theta t_{1}}\right)^{\alpha+\beta-1}} \int_{t_{1}}^{t_{2}}(1+\theta x)^{-2(\alpha+\beta-1)} d x\right)\right] \\
& =\frac{1}{\beta-\alpha}\left[\log \left(\frac{1}{\left(\frac{t_{2}}{1+\theta t_{2}}-\frac{t_{1}}{1+\theta t_{1}}\right)^{\alpha+\beta-1}} \times \frac{\left(1+\theta t_{2}\right)^{1-2(\alpha+\beta-1)}-\left(1+\theta t_{1}\right)^{1-2(\alpha+\beta-1)}}{1-2(\alpha+\beta-1)}\right)\right] .
\end{aligned}
$$




\section{Characterization Results Based on $H_{\alpha}^{\beta}\left(X ; t_{1}, t_{2}\right)$}

In this section we show that $H_{\alpha}^{\beta}\left(X ; t_{1}, t_{2}\right)$ uniquely determines the lifetime distribution function. In this context we prove the following result. We first give the definition of general failure rate (GFR) functions, refer to Navarro and Ruiz (1996, 2004).

\section{Definition 1}

The GFR functions of a doubly truncated random variable $\left(X \mid t_{1} \leq X \leq t_{2}\right)$ are given by $h_{1}^{X}\left(t_{1}, t_{2}\right)=\frac{f\left(t_{1}\right)}{F\left(t_{2}\right)-F\left(t_{1}\right)}$ and $h_{2}^{X}\left(t_{1}, t_{2}\right)=\frac{f\left(t_{2}\right)}{F\left(t_{2}\right)-F\left(t_{1}\right)}$.

\section{Theorem 2}

Let $X$ be a non-negative random variable having continuous density function $f(x)$ and distribution function $F(x)$. Assume that $H_{\alpha}^{\beta}\left(X ; t_{1}, t_{2}\right)$ be increasing with respect to both coordinates $t_{1}$ and $t_{2}$. Then for each $\alpha$ and $\beta$, $H_{\alpha}^{\beta}\left(X ; t_{1}, t_{2}\right)$ uniquely determines the distribution function $F(x)$.

Proof On differentiating (6) with respect to $t_{1}$ and $t_{2}$, we obtain

$$
\begin{aligned}
h_{2}^{\alpha+\beta-1}\left(t_{1}, t_{2}\right)=(\alpha+\beta & -1) \exp \left\{(\beta-\alpha) H_{\alpha}^{\beta}\left(X ; t_{1}, t_{2}\right)\right\} h_{2}\left(t_{1}, t_{2}\right) \\
& +(\beta-\alpha) \exp \left\{(\beta-\alpha) H_{\alpha}^{\beta}\left(X ; t_{1}, t_{2}\right)\right\} \frac{\partial}{\partial t_{2}} H_{\alpha}^{\beta}\left(X ; t_{1}, t_{2}\right),
\end{aligned}
$$

and

$$
\begin{aligned}
& h_{1}^{\alpha+\beta-1}\left(t_{1}, t_{2}\right)=(\alpha+\beta-1) \exp \left\{(\beta-\alpha) H_{\alpha}^{\beta}\left(X ; t_{1}, t_{2}\right)\right\} h_{1}\left(t_{1}, t_{2}\right) \\
&-(\beta-\alpha) \exp \left\{(\beta-\alpha) H_{\alpha}^{\beta}\left(X ; t_{1}, t_{2}\right)\right\} \frac{\partial}{\partial t_{1}} H_{\alpha}^{\beta}\left(X ; t_{1}, t_{2}\right) .
\end{aligned}
$$

Hence for fixed and positive $t_{1}$ and $t_{2}, h_{1}\left(t_{1}, t_{2}\right)$ and $h_{2}\left(t_{1}, t_{2}\right)$ are solutions of $g\left(x_{t_{2}}\right)=0$ and $k\left(y_{t_{1}}\right)=0$, where

$$
\begin{aligned}
g\left(x_{t_{2}}\right)=x_{t_{2}}^{\alpha+\beta-1}- & (\alpha+\beta-1) x_{t_{2}} \exp \left\{(\beta-\alpha) H_{\alpha}^{\beta}\left(X ; t_{1}, t_{2}\right)\right\} \\
& -(\beta-\alpha) \exp \left\{(\beta-\alpha) H_{\alpha}^{\beta}\left(X ; t_{1}, t_{2}\right)\right\} \frac{\partial}{\partial t_{2}} H_{\alpha}^{\beta}\left(X ; t_{1}, t_{2}\right),
\end{aligned}
$$

and

$$
\begin{aligned}
k\left(y_{t_{1}}\right)=y_{t_{1}}^{\alpha+\beta-1}- & (\alpha+\beta-1) y_{t_{1}} \exp \left\{(\beta-\alpha) H_{\alpha}^{\beta}\left(X ; t_{1}, t_{2}\right)\right\} \\
& +(\beta-\alpha) \exp \left\{(\beta-\alpha) H_{\alpha}^{\beta}\left(X ; t_{1}, t_{2}\right)\right\} \frac{\partial}{\partial t_{1}} H_{\alpha}^{\beta}\left(X ; t_{1}, t_{2}\right) .
\end{aligned}
$$

Partial differentiating (15) and (16) with respect to $x_{t_{2}}$ and $y_{t_{1}}$, we obtain

$$
\frac{\partial g\left(x_{t_{2}}\right)}{\partial x_{t_{2}}}=(\alpha+\beta-1) x_{t_{2}}^{\alpha+\beta-2}-(\alpha+\beta-1) \exp \left\{(\beta-\alpha) H_{\alpha}^{\beta}\left(X ; t_{1}, t_{2}\right)\right\},
$$

and

$$
\frac{\partial k\left(y_{t_{1}}\right)}{\partial y_{t_{1}}}=(\alpha+\beta-1) y_{t_{1}}^{\alpha+\beta-2}-(\alpha+\beta-1) \exp \left\{(\beta-\alpha) H_{\alpha}^{\beta}\left(X ; t_{1}, t_{2}\right)\right\} .
$$

For extreme value of $g\left(x_{t_{2}}\right)$ and $k\left(y_{t_{1}}\right)$, we have $\frac{\partial g\left(x_{t_{2}}\right)}{\partial x_{t_{2}}}=0$ and $\frac{\partial k\left(y_{t_{1}}\right)}{\partial y_{t_{1}}}=0$, which gives as $x_{t_{2}}=$ $\left(\exp \left\{(\beta-\alpha) H_{\alpha}^{\beta}\left(X ; t_{1}, t_{2}\right)\right\}\right)^{\frac{1}{\alpha+\beta-2}}=y_{t_{1}}$, respectively. Furthermore, second order derivatives are

$$
\frac{\partial^{2} g\left(x_{t_{2}}\right)}{\partial x_{t_{2}}^{2}}=(\alpha+\beta-1)(\alpha+\beta-2) x_{t_{2}}^{\alpha+\beta-3}
$$


and

$$
\frac{\partial^{2} k\left(y_{t_{1}}\right)}{\partial y_{t_{1}}^{2}}=(\alpha+\beta-1)(\alpha+\beta-2) y_{t_{1}}^{\alpha+\beta-3}
$$

Case I: Let $\alpha+\beta>2$, then $\frac{\partial^{2} g\left(x_{t_{2}}\right)}{\partial x_{t_{2}}^{2}}=\frac{\partial^{2} k\left(y_{t_{1}}\right)}{\partial y_{t_{1}}^{2}}>0$. Thus both of $g\left(x_{t_{2}}\right)$ and $k\left(y_{t_{1}}\right)$ are minimized at $x_{t_{2}}$ and $y_{t_{1}}$ respectively. Also, $g(0)=-(\beta-\alpha) \exp \left\{(\beta-\alpha) H_{\alpha}^{\beta}\left(X ; t_{1}, t_{2}\right)\right\} \frac{\partial}{\partial t_{2}} H_{\alpha}^{\beta}\left(X ; t_{1}, t_{2}\right)<0$, since we assume that $H_{\alpha}^{\beta}\left(X ; t_{1}, t_{2}\right)$ be increasing with respect to both coordinates $t_{1}$ and $t_{2}$, and $g(\infty)=\infty$. Similarly $k(0)>0$ and $k(\infty)=\infty$. Therefore, both the equations $g\left(x_{t_{2}}\right)=0$ and $k\left(y_{t_{1}}\right)=0$ have unique positive solutions $h_{1}\left(t_{1}, t_{2}\right)$ and $h_{2}\left(t_{1}, t_{2}\right)$ respectively.

Case II: Let $\alpha+\beta<2$, then $\frac{\partial^{2} g\left(x_{t_{2}}\right)}{\partial x_{t_{2}}^{2}}=\frac{\partial^{2} k\left(y_{t_{1}}\right)}{\partial y_{t_{1}}^{2}}<0$. Thus both of $g\left(x_{t_{2}}\right)$ and $k\left(y_{t_{1}}\right)$ are maximized at $x_{t_{2}}$ and $y_{t_{1}}$ respectively. In the same way one can conclude that $h_{1}\left(t_{1}, t_{2}\right)$ and $h_{2}\left(t_{1}, t_{2}\right)$ are unique solutions of $g\left(x_{t_{2}}\right)=0$, and $k\left(y_{t_{1}}\right)=0$ respectively. So, $H_{\alpha}^{\beta}\left(X ; t_{1}, t_{2}\right)$ determines the generalized failure rates $h_{j}\left(t_{1}, t_{2}\right), j=1,2$ uniquely.

The characterization of specific distributions using relations between reliability measures has become of increasing interest. Next we characterize uniform distribution in term of the generalized interval entropy (5). We give the following theorem.

Theorem 3

A random variable $X$ over $(a, b), a<b$, has uniform distribution if, and only if

$$
H_{\alpha}^{\beta}\left(X ; t_{1}, t_{2}\right)=\left(\frac{2-\alpha-\beta}{\beta-\alpha}\right) \log \left(t_{2}-t_{1}\right) .
$$

Proof The 'only if'; part of the theorem is straight forward. Since in case of uniform distribution of $X$ over $(a, b)$

$$
F(x)=\frac{x-a}{b-a} \text { and } f(x)=\frac{1}{b-a} .
$$

Substituting these in (5) and simplifying, we obtain

$$
H_{\alpha}^{\beta}\left(X ; t_{1}, t_{2}\right)=\left(\frac{2-\alpha-\beta}{\beta-\alpha}\right) \log \left(t_{2}-t_{1}\right) .
$$

To prove the 'if part' let (19) be valid. Which gives

$$
\int_{t_{1}}^{t_{2}} f^{\alpha+\beta-1}(x) d x=\left(t_{2}-t_{1}\right)^{2-\alpha-\beta}\left[F\left(t_{2}\right)-F\left(t_{1}\right)\right]^{\alpha+\beta-1} .
$$

Differentiating (20) with respect to $t_{1}$ and $t_{2}$, we obtain

$$
h_{1}^{\alpha+\beta-1}\left(t_{1}, t_{2}\right)=\frac{(2-\alpha-\beta)}{\left(t_{2}-t_{1}\right)^{\alpha+\beta-1}}+(\alpha+\beta-1) h_{1}\left(t_{1}, t_{2}\right)\left(t_{2}-t_{1}\right)^{2-\alpha-\beta}
$$

and

$$
h_{2}^{\alpha+\beta-1}\left(t_{1}, t_{2}\right)=\frac{(2-\alpha-\beta)}{\left(t_{2}-t_{1}\right)^{\alpha+\beta-1}}+(\alpha+\beta-1) h_{2}\left(t_{1}, t_{2}\right)\left(t_{2}-t_{1}\right)^{2-\alpha-\beta} .
$$

Then, for any fixed $t_{1}$ and arbitrary $t_{2}, h_{1}^{X}\left(t_{1}, t_{2}\right)$ is a positive solution of $\eta\left(x_{t_{2}}\right)=0$, where

$$
\eta\left(x_{t_{2}}\right)=\left\{x_{t_{2}}\right\}^{\alpha+\beta-1}-(\alpha+\beta-1) x_{t_{2}}\left(t_{2}-t_{1}\right)^{2-\alpha+\beta}-\left\{\frac{2-\alpha+\beta}{\left(t_{2}-t_{1}\right)^{\alpha+\beta-1}}\right\} .
$$


Similarly, for any fixed $t_{2}$ and arbitrary $t_{1}, h_{2}^{X}\left(t_{1}, t_{2}\right)$ is a positive solution of $\zeta\left(y_{t_{1}}\right)=0$, where

$$
\zeta\left(y_{t_{1}}\right)=\left\{y_{t_{1}}\right\}^{\alpha+\beta-1}-(\alpha+\beta-1) y_{t_{1}}\left(t_{2}-t_{1}\right)^{2-\alpha+\beta}-\left\{\frac{2-\alpha+\beta}{\left(t_{2}-t_{1}\right)^{\alpha+\beta-1}}\right\} .
$$

Differentiating both side of (21) and (22) with respect to $x_{t_{2}}$ and $y_{t_{1}}$ respectively, we get

$$
h_{1}^{X}\left(t_{1}, t_{2}\right)=h_{2}^{X}\left(t_{1}, t_{2}\right)=\frac{1}{t_{2}-t_{1}}
$$

Thus, if lifetime of a component is uniformly distributed in interval $(a, b)$, then the generalized failure rate is, $h_{i}^{X}\left(t_{1}, t_{2}\right)=\frac{1}{t_{2}-t_{1}} ;$ for $i=1,2$. Thus result is prove.

\section{Lifetime Distributions Based on Interval Entropy of Order $\alpha$ and Type $\beta$}

In this section we characterize some specific lifetime distribution functions based on $H_{\alpha}^{\beta}\left(X ; t_{1}, t_{2}\right)$, the generalized interval entropy of order $\alpha$ and type $\beta$. We will achieve this by considering a relation between $H_{\alpha}^{\beta}\left(X ; t_{1}, t_{2}\right)$ and $h_{i}^{X}\left(t_{1}, t_{2}\right)$ for $i=1,2$, the general failure rate (GFR) function. We give the following result.

\section{Theorem 4}

Let $X$ be a non-negative continuous random variable with survival function $\bar{F}(t)$, general failure rate function $h_{i}^{X}\left(t_{1}, t_{2}\right)$ for $i=1,2$ and generalized interval entropy $H_{\alpha}^{\beta}\left(X ; t_{1}, t_{2}\right)$ given by

$$
(\beta-\alpha) H_{\alpha}^{\beta}\left(X ; t_{1}, t_{2}\right)=\log \left\{\frac{1}{k}\left[\left(1+c t_{2}\right) h_{2}^{\alpha+\beta-1}\left(t_{1}, t_{2}\right)-\left(1+c t_{1}\right) h_{1}^{\alpha+\beta-1}\left(t_{1}, t_{2}\right)\right]\right\},
$$

where $k$ is constant hold for all $\left(t_{1}, t_{2}\right) \in D$. Then $X$ has (i) an exponential distribution iff $c=0$, (ii) a Pareto distribution iff $c<0$, and (iii) a finite range distribution iff $c>0$.

Proof (i) The p.d.f. and survival function of an exponential variable $X$ with parameter $\theta>0$, are given respectively by

$$
f(x)=\theta e^{-\theta x} \text { and } \bar{F}(x)=e^{-\theta x}
$$

The general failure rate functions are

$$
h_{i}\left(t_{1}, t_{2}\right)=\frac{f\left(t_{i}\right)}{F\left(t_{2}\right)-F\left(t_{1}\right)}=\frac{\theta e^{-\theta t_{i}}}{e^{-\theta t_{1}}-e^{-\theta t_{2}}}, i=1,2 .
$$

The generalized interval entropy $H_{\alpha}^{\beta}\left(X ; t_{1}, t_{2}\right)$ is given by

$$
\begin{aligned}
(\beta-\alpha) H_{\alpha}^{\beta}(X ; & \left.t_{1}, t_{2}\right)=\log \left[\int_{t_{1}}^{t_{2}}\left(\frac{f(x)}{F\left(t_{2}\right)-F\left(t_{1}\right)}\right)^{\alpha+\beta-1} d x\right] \\
= & \log \left[\int_{t_{1}}^{t_{2}}\left(\frac{\theta e^{-\theta x}}{e^{-\theta t_{1}}-e^{-\theta t_{2}}}\right)^{\alpha+\beta-1} d x\right] \\
= & \log \left\{\frac{\theta^{\alpha+\beta-1}}{-(\alpha+\beta-1) \theta} \frac{\left[e^{-(\alpha+\beta-1) \theta t_{2}}-e^{-(\alpha+\beta-1) \theta t_{1}}\right]}{\left(e^{-\theta t_{1}}-e^{-\theta t_{2}}\right)^{\alpha+\beta-1}}\right\} \\
= & \log \left\{\frac{1}{k}\left[h_{2}^{\alpha+\beta-1}\left(t_{1}, t_{2}\right)-h_{1}^{\alpha+\beta-1}\left(t_{1}, t_{2}\right)\right]\right\},
\end{aligned}
$$


which is (24) for $c=0$.

(ii) The p.d.f. of the pareto distribution is given by

$$
f(x)=p q(1+p x)^{-(q+1)}, \quad p, q>0
$$

and the survival function is

$$
\bar{F}(x)=1-F(x)=(1+p x)^{-q} .
$$

The general failure rate functions are

$$
h_{i}\left(t_{1}, t_{2}\right)=\frac{f\left(t_{i}\right)}{F\left(t_{2}\right)-F\left(t_{1}\right)}=\frac{p q\left(1+p t_{i}\right)^{-(q+1)}}{\left(1+p t_{1}\right)^{-q}-\left(1+p t_{2}\right)^{-q}}, i=1,2 .
$$

Substituting in (5) and simplifying, we obtain

$$
\begin{aligned}
(\beta-\alpha) H_{\alpha}^{\beta}\left(X ; t_{1}, t_{2}\right)= & \log \left\{\frac{(p q)^{\alpha+\beta-1}}{p[1-(q+1)(\alpha+\beta-1)]} \times\right. \\
& \left.\frac{\left[\left(1+p t_{2}\right)^{1-(\alpha+\beta-1)(q+1)}-\left(1+p t_{1}\right)^{1-(\alpha+\beta-1)(q+1)}\right]}{\left\{\left(1+p t_{1}\right)^{-q}-\left(1+p t_{2}\right)^{-q}\right\}^{\alpha+\beta-1}}\right\} .
\end{aligned}
$$

It can be rewritten as

$$
(\beta-\alpha) H_{\alpha}^{\beta}\left(X ; t_{1}, t_{2}\right)=\log \left\{\frac{1}{k}\left[\left(1+p t_{2}\right) h_{2}^{\alpha+\beta-1}\left(t_{1}, t_{2}\right)-\left(1+p t_{1}\right) h_{1}^{\alpha+\beta-1}\left(t_{1}, t_{2}\right)\right]\right\},
$$

where $k=p[1-(q+1)(\alpha+\beta-1)]$ and $c=p>0$. Thus (24) holds.

(iii) The p.d.f. and survival function of the finite range distribution are given respectively by

$$
f(x)=a b(1-a x)^{b-1}, \quad a, b>0, \text { and } 0 \leq x \leq \frac{1}{a},
$$

and

$$
\bar{F}(x)=1-F(x)=(1-a x)^{b} .
$$

The general failure rate functions are

$$
h_{i}\left(t_{1}, t_{2}\right)=\frac{f\left(t_{i}\right)}{F\left(t_{2}\right)-F\left(t_{1}\right)}=\frac{a b\left(1-a t_{i}\right)^{b-1}}{\left(1-a t_{1}\right)^{b}-\left(1-a t_{2}\right)^{b}}, i=1,2 .
$$

Substituting in (5) and simplifying, we obtain

$$
\begin{aligned}
(\beta-\alpha) H_{\alpha}^{\beta}\left(X ; t_{1}, t_{2}\right)= & \log \left\{\frac{(a b)^{\alpha+\beta-1}}{-a[1+(b-1)(\alpha+\beta-1)]} \times\right. \\
& \left.\frac{\left[\left(1-a t_{2}\right)^{1+(\alpha+\beta-1)(b-1)}-\left(1-a t_{1}\right)^{1+(\alpha+\beta-1)(b-1)}\right]}{\left\{\left(1-a t_{1}\right)^{b}-\left(1-a t_{2}\right)^{b}\right\}^{\alpha+\beta-1}}\right\} .
\end{aligned}
$$

This gives

$$
(\beta-\alpha) H_{\alpha}^{\beta}\left(X ; t_{1}, t_{2}\right)=\log \left\{\frac{1}{k}\left[\left(1-a t_{2}\right) h_{1}^{\alpha+\beta-1}\left(t_{1}, t_{2}\right)-\left(1-a t_{1}\right) h_{2}^{\alpha+\beta-1}\left(t_{1}, t_{2}\right)\right]\right\},
$$

where $k=-a[1+(b-1)(\alpha+\beta-1)]$ and $c=-a<0$. Thus (24) holds.

Conversely, assume that (24) is valid. Using (5) in (24) and simplifying, we get

$$
k \int_{t_{1}}^{t_{2}} f^{\alpha+\beta-1}(x) d x=\left(1+c t_{2}\right) f^{\alpha+\beta-1}\left(t_{2}\right)-\left(1+c t_{1}\right) f^{\alpha+\beta-1}\left(t_{1}\right) .
$$


Differentiate (30) with respect to $t_{2}$ keeping $t_{1}$ is fixed, we get

$$
k f^{\alpha+\beta-1}\left(t_{2}\right)=(\alpha+\beta-1)\left(1+c t_{2}\right) f^{\alpha+\beta-2}\left(t_{2}\right) f^{\prime}\left(t_{2}\right)+c f^{\alpha+\beta-1}\left(t_{2}\right) .
$$

Dividing by $f^{\alpha+\beta-1}\left(t_{2}\right)$ both side and simplify, we obtain

$$
\frac{f^{\prime}\left(t_{2}\right)}{f\left(t_{2}\right)}=\left(\frac{k-c}{\alpha+\beta-1}\right) \frac{1}{1+c t_{2}} .
$$

Similarly, we get a relation differentiating with respect to $t_{1}$, as $t_{2}$ is fixed. Thus, finally we get

$$
\frac{f^{\prime}\left(t_{i}\right)}{f\left(t_{i}\right)}=\left(\frac{k-c}{\alpha+\beta-1}\right) \frac{1}{1+c t_{i}} \text { for }\left(t_{1}, t_{2}\right) \in D \quad i=1,2 .
$$

This gives

$$
\frac{d}{d t} \log f\left(t_{i}\right)=\left(\frac{k-c}{\alpha+\beta-1}\right) \frac{1}{1+c t_{i}}, i=1,2 .
$$

The Eq. (32) represents the underlying distribution is exponential if $c=0$, Pareto distribution for $c>0$, and finite range distribution for $c<0$. This proves the theorem.

\subsection{A Lower Bound for $H_{\alpha}^{\beta}\left(X ; t_{1}, t_{2}\right)$}

In the following theorem we provide lower bound for the generalized interval entropy measure of order $\alpha$ and type $\beta$ based on monotonic behavior of the general failure rate (GFR) of the doubly truncated random variable.

\section{Theorem 5}

Let $X$ be an absolutely continuous random variable with density $f(x)$ and cumulative distribution function $F(x)$. (i) If $h_{1}^{X}\left(t_{1}, t_{2}\right)$ is increasing in $t_{1}$, then

$$
H_{\alpha}^{\beta}\left(X ; t_{1}, t_{2}\right) \geq \frac{1}{\beta-\alpha} \log h_{1}^{\alpha+\beta-1}\left(t_{1}, t_{2}\right),
$$

and (ii) If $h_{2}^{X}\left(t_{1}, t_{2}\right)$ is decreasing in $t_{2}$, then

$$
H_{\alpha}^{\beta}\left(X ; t_{1}, t_{2}\right) \geq \frac{1}{\beta-\alpha} \log h_{1}^{\alpha+\beta-1}\left(t_{1}, t_{2}\right)
$$

Proof From (5), we have

$$
\begin{aligned}
& H_{\alpha}^{\beta}\left(X ; t_{1}, t_{2}\right)=\frac{1}{\beta-\alpha} \log \left[\int_{t_{1}}^{t_{2}}\left(\frac{f(x)}{F\left(t_{2}\right)-F\left(t_{1}\right)}\right)^{\alpha+\beta-1} d x\right] \\
& =\frac{1}{\beta-\alpha} \log \left[\int_{t_{1}}^{t_{2}} h_{1}^{\alpha+\beta-1}\left(x, t_{2}\right)\left(\frac{F\left(t_{2}\right)-F(x)}{F\left(t_{2}\right)-F\left(t_{1}\right)}\right)^{\alpha+\beta-1} d x\right] .
\end{aligned}
$$

Since $\frac{F\left(t_{2}\right)-F(x)}{F\left(t_{2}\right)-F\left(t_{1}\right)} \geq 0$ for $t_{1}<x$, and by the assumption that GFR $h_{1}^{X}\left(t_{1}, t_{2}\right)$ is increasing in $t_{1}$, we have $h_{1}^{X}\left(x, t_{2}\right) \geq h_{1}^{X}\left(t_{1}, t_{2}\right)$, thus

$$
\begin{aligned}
& H_{\alpha}^{\beta}\left(X ; t_{1}, t_{2}\right) \geq \frac{1}{\beta-\alpha} \log \left[\int_{t_{1}}^{t_{2}} h_{1}^{\alpha+\beta-1}\left(t_{1}, t_{2}\right)\left(\frac{F\left(t_{2}\right)-F(x)}{F\left(t_{2}\right)-F\left(t_{1}\right)}\right)^{\alpha+\beta-1} d x\right], \\
& =\frac{1}{\beta-\alpha}\left[\log h_{1}^{\alpha+\beta-1}\left(t_{1}, t_{2}\right)+\log \int_{t_{1}}^{t_{2}}\left(\frac{F\left(t_{2}\right)-F(x)}{F\left(t_{2}\right)-F\left(t_{1}\right)}\right)^{\alpha+\beta-1} d x\right], \\
& \geq \frac{1}{\beta-\alpha} \log h_{1}^{\alpha+\beta-1}\left(t_{1}, t_{2}\right) .
\end{aligned}
$$

The proof of the second part is similar to that of (i), using $h_{2}^{X}\left(t_{1}, x\right) \leq h_{2}^{X}\left(t_{1}, t_{2}\right)$ for $t_{2}>x$. Hence omitted. This completes the proof. 


\section{Stochastic Comparison Based on Generalized Interval Entropy}

Next, we present some stochastic comparison results as follows: Let $X$ and $Y$ be two absolutely continuous random variables with distribution functions $F$ and $G$. Let $\bar{F}$ and $\bar{G}$ denote their survival functions with probability density functions $f$ and $g$, respectively. Then

(i) A random variable $X$ is said to be less than or equal to $Y$ in dispersion ordering (denoted by $X \leq{ }^{d} Y$, if and only if

$$
g\left(G^{-1}(u)\right) \leq f\left(F^{-1}(u)\right), \quad \forall 0 \leq v \leq u \leq 1 .
$$

(ii) Let $X$ and $Y$ be two non-negative random variables. Then $X$ is said to be smaller than $Y$ in residual entropy order (denoted as $X \leq{ }^{R E} Y$ ) if $H(X ; t) \leq H(Y ; t)$ for all $t \geq 0$.

(iii) Let $X$ and $Y$ be two non-negative random variables. Then $X$ is said to be smaller than $Y$ in past entropy order (denoted as $X \leq^{P E} Y$ ) if $\bar{H}(X ; t) \leq \bar{H}(Y ; t)$ for all $t \geq 0$.

For more details refer to Shaked and Shanthikumar [18], Ebrahimi and Pellerey [9] and, Nanda and Paul [15]. Ebrahimi [8] also introduced two new non-parametric classes of life distributions via monotonicity properties of the uncertainly residual (past) lifetime function.

\section{Definition 2}

A random variable $X$ is said to have decreasing (increasing) uncertainty of residual life (DURL (IURL)), if $H(X ; t)$ is decreasing (increasing) in $t \geq 0$.

\section{Definition 3}

A random variable $X$ is said to have decreasing (increasing) uncertainty of past life (DUPL (IUPL)), if $\bar{H}(X ; t)$ is decreasing (increasing) in $t \geq 0$.

The following stochastic orders based on generalized interval entropy of order $\alpha$ and type $\beta$ could be defined similarly.

A random variable $X$ is said to be less than or equal to $Y$ in Verma's entropy ordering (denoted by $X \leq{ }^{V e} Y$ ) if $H_{\alpha}^{\beta}(X) \leq H_{\alpha}^{\beta}(Y), \forall \beta-1<\alpha<\beta, \beta \geq 1$.

\section{Theorem 6}

If $X$ and $Y$ are two random variables such that $X \leq^{d} Y$, then $H_{\alpha}^{\beta}(X) \leq H_{\alpha}^{\beta}(Y)$.

Proof The probability integral transformation provides the following useful representation of the Verma's entropy measure (2) for the random variable $X$

$$
H_{\alpha}^{\beta}(X)=\frac{1}{\beta-\alpha} \log \left\{\int_{0}^{1} f^{\alpha+\beta-2}\left(F^{-1}(u)\right) d u\right\} .
$$

Since $X \leq^{d} Y$ we have

$$
\begin{aligned}
& \int_{0}^{1}\left\{f^{\alpha+\beta-2}\left(F^{-1}(u)\right)-g^{\alpha+\beta-2}\left(G^{-1}(u)\right)\right\} d u \geq 0, \quad \forall \quad \alpha+\beta>2, \\
& \int_{0}^{1}\left\{f^{\alpha+\beta-2}\left(F^{-1}(u)\right)-g^{\alpha+\beta-2}\left(G^{-1}(u)\right)\right\} d u \leq 0, \quad \forall \quad \alpha+\beta<2,
\end{aligned}
$$

since $\log$ is an increasing function, thus result follows.

\section{Definition 4}

A distribution function $F($.$) has decreasing (or, increasing) generalized interval entropy (DGIE (\alpha, \beta)$ )(or, IGIE $(\alpha, \beta)$ ), if $H_{\alpha}^{\prime \beta}\left(Y ; t_{1}, t_{2}\right)$ is decreasing (increasing) in $t_{1}$, for fixed $t_{2}$.

Definition 5

A distribution function $F($.$) has decreasing (or, increasing) generalized interval entropy (DGIE (\alpha, \beta)$ )(or, IGIE $(\alpha, \beta))$, if $H_{\alpha}^{\prime \beta}\left(Y ; t_{1}, t_{2}\right)$ is decreasing in $t_{2}$, for fixed $t_{1}$. 
In many cases of practical interest are would like to know whether the DGIE (IGIE) property of $X$ is inherited by a transformation of $X$. The following Theorem provides a partial answer.

\section{Theorem 7}

(a) If $X$ is IGIE $(\alpha, \beta)$ and if $\phi$ is non negative, increasing and convex, then $\phi(X)$ is also IGIE $(\alpha, \beta)$.

(b) If $X$ is DGIE $(\alpha, \beta)$ and if $\phi$ is non negative, increasing and concave, then $\phi(X)$ is also DGIE $(\alpha, \beta)$.

Proof From (10), we have

$$
H_{\alpha}^{\beta}\left(Y ; t_{1}, t_{2}\right)=\frac{1}{\beta-\alpha} \log \left[\int_{\phi^{-1}\left(t_{1}\right)}^{\phi^{-1}\left(t_{2}\right)}\left(\frac{f(x)}{F\left(\phi^{-1}\left(t_{2}\right)\right)-F\left(\phi^{-1}\left(t_{1}\right)\right)}\right)^{\alpha+\beta-1}\left(\frac{1}{\phi^{\prime}(x)}\right)^{\alpha+\beta-2} d x\right] .
$$

This can be written as

$$
\begin{aligned}
(\beta-\alpha) H_{\alpha}^{\beta}\left(Y ; t_{1}, t_{2}\right)=(1-\alpha-\beta) \log [ & \left.F\left(\phi^{-1}\left(t_{2}\right)\right)-F\left(\phi^{-1}\left(t_{1}\right)\right)\right] \\
+ & \log \left[\int_{\phi^{-1}\left(t_{1}\right)}^{\phi^{-1}\left(t_{2}\right)} f(x)^{\alpha+\beta-1} \phi^{\prime}(x)^{2-\alpha-\beta} d x\right] .
\end{aligned}
$$

On differentiating (35) with respect to $t_{1}$, keeping $t_{2}$ is fixed, we get

$$
\begin{aligned}
&(\beta-\alpha) \frac{\partial H_{\alpha}^{\beta}\left(Y ; t_{1}, t_{2}\right)}{\partial t_{1}}=\frac{(\alpha+\beta-1) f\left(\phi^{-1}\left(t_{1}\right)\right)}{\left[F\left(\phi^{-1}\left(t_{2}\right)\right)-F\left(\phi^{-1}\left(t_{1}\right)\right)\right] \phi^{\prime}\left(t_{1}\right)} \\
&-\frac{f^{\alpha+\beta-1}\left(\phi^{-1}\left(t_{1}\right)\right)\left[\phi^{\prime}\left(\phi^{-1}\left(t_{1}\right)\right)\right]^{2-\alpha-\beta}}{\left[\int_{\phi^{-1}\left(t_{1}\right)}^{\phi^{-1}\left(t_{2}\right)} f(x)^{\alpha+\beta-1}\left[\phi^{\prime}(x)\right]^{2-\alpha-\beta} d x\right]},
\end{aligned}
$$

or,

$$
\begin{gathered}
=\frac{(\alpha+\beta-1) h_{1, \phi}\left(\phi^{-1}\left(t_{1}\right), \phi^{-1}\left(t_{2}\right)\right)}{\phi^{\prime}\left(t_{1}\right)} \\
-\frac{\left[h_{1, \phi}\left(\phi^{-1}\left(t_{1}\right), \phi^{-1}\left(t_{2}\right)\right)\right]^{\alpha+\beta-1}}{\phi^{\prime}\left(t_{1}\right)} \cdot \frac{\left[\phi^{\prime}\left(\phi^{-1}\left(t_{1}\right)\right)\right]^{2-\alpha-\beta}}{\int_{\phi^{-1}\left(t_{1}\right)}^{\phi^{-1}\left(t_{2}\right)}\left(\frac{f(x)}{F\left(\phi^{-1}\left(t_{2}\right)\right)-F\left(\phi^{-1}\left(t_{1}\right)\right)}\right)^{\alpha+\beta-1}\left[\phi^{\prime}(x)\right]^{2-\alpha-\beta} d x},
\end{gathered}
$$

where $h_{1, \phi}\left(\phi^{-1}\left(t_{1}\right), \phi^{-1}\left(t_{2}\right)\right)=\frac{f\left(\phi^{-1}\left(t_{1}\right)\right)}{F\left(\phi^{-1}\left(t_{2}\right)\right)-F\left(\phi^{-1}\left(t_{1}\right)\right)}$ is the general failure rate (GFR) function of the doubly truncated random variable $\left(X \mid \phi^{-1}\left(t_{1}\right) \leq X \leq \phi^{-1}\left(t_{2}\right)\right)$. Let $\alpha+\beta>2, \phi^{\prime}(x)$ is an increasing function because $\phi(x)$ is a convex function. So $\phi^{\prime(2-\alpha-\beta)}(x)$ is a decreasing function, that is

$$
\left[\phi^{\prime}(x)\right]^{2-\alpha-\beta} \leq\left[\phi^{\prime}\left(\phi^{-1}\left(t_{1}\right)\right)\right]^{2-\alpha-\beta}, \quad \forall x>\phi^{-1}\left(t_{1}\right) .
$$

Hence,

$$
\begin{gathered}
\frac{\partial H_{\alpha}^{\beta}\left(Y ; t_{1}, t_{2}\right)}{\partial t_{1}} \geq \frac{(\alpha+\beta-1) h_{1, \phi}\left(\phi^{-1}\left(t_{1}\right), \phi^{-1}\left(t_{2}\right)\right)}{(\beta-\alpha) \phi^{\prime}\left(t_{1}\right)} \\
-\frac{\left[h_{1, \phi}\left(\phi^{-1}\left(t_{1}\right), \phi^{-1}\left(t_{2}\right)\right)\right]^{\alpha+\beta-1}}{(\beta-\alpha) \phi^{\prime}\left(t_{1}\right)} \cdot \frac{1}{\int_{\phi^{-1}\left(t_{1}\right)}^{\phi^{-1}\left(t_{2}\right)}\left(\frac{f(x)}{F\left(\phi^{-1}\left(t_{2}\right)\right)-F\left(\phi^{-1}\left(t_{1}\right)\right)}\right)^{\alpha+\beta-1} d x}, \\
=\frac{1}{\phi^{\prime}\left(t_{1}\right)}\left\{\frac{(\alpha+\beta-1)}{(\beta-\alpha)} h_{1, \phi}\left(\phi^{-1}\left(t_{1}\right), \phi^{-1}\left(t_{2}\right)\right)\right. \\
\left.-\frac{\left[h_{1, \phi}\left(\phi^{-1}\left(t_{1}\right), \phi^{-1}\left(t_{2}\right)\right)\right]^{\alpha+\beta-1}}{(\beta-\alpha)} e^{(\alpha-\beta) H_{\alpha}^{\beta}\left(X ; \phi^{-1}\left(t_{1}\right), \phi^{-1}\left(t_{2}\right)\right)}\right\} .
\end{gathered}
$$




$$
=\frac{1}{\phi^{\prime}\left(t_{1}\right)}\left[H_{\alpha}^{\prime \beta}\left(X ; \phi^{-1}\left(t_{1}\right), \phi^{-1}\left(t_{2}\right)\right)\right] \geq 0 .
$$

Similarly on differentiating (35) with respect to $t_{2}$, keeping $t_{1}$ is fixed, we get

$$
\frac{\partial H_{\alpha}^{\beta}\left(Y ; t_{1}, t_{2}\right)}{\partial t_{2}}=\frac{1}{\phi^{\prime}\left(t_{2}\right)}\left[H_{\alpha}^{\prime \beta}\left(X ; \phi^{-1}\left(t_{1}\right), \phi^{-1}\left(t_{2}\right)\right)\right] \geq 0 .
$$

A similar result follow for $\alpha+\beta<2$.

(b) The proof is similar to that of (a), hence omitted.

\section{Conclusion}

When a system has survived only between two time points $\left(t_{1}, t_{2}\right)$, generalized interval entropy plays an important role in the field of information theory and survival analysis. We discussed the generalized interval entropy measure associated with various distributions, which play a vital role in reliability modeling. Also we characterize some specific lifetime distribution using the relationship between proposed entropy $H_{\alpha}^{\beta}\left(X ; t_{1}, t_{2}\right)$, and reliability measure. The characterizations, stochastic ordering and other properties obtained here prove the interest of these concepts in measuring the uncertainty contained in a doubly truncated random variable. Also the results reported generalize the existing results in context with interval entropy.

\section{Acknowledgement}

The authors would like to express their gratitude to the reviewers and the editor-in-chief for their valuable comments, which have considerably improved the earlier version of the article.

\section{REFERENCES}

1. M. Asadi, N. Ebrahimi, and E. Soofi, Dynamic generalized information measures, Statistics and Probability Letters, vol. 71, no. 1, pp. 85-98, 2005.

2. M. Asadi, and N. Ebrahimi, Residual entropy and its characterizations in terms of hazard function and mean residual life function, Statistics and Probability Letters, vol. 49, no. 3, pp. 263-269, 2007.

3. T. Azlarov, and N. Volodin, Characterization problems associated with exponential distribution, Springer-Verlag, New York, 1986.

4. M. A. K. Baig, and J. G. Dar, Generalized residual entropy function and its applications, Europian Journal of Pure and Applied Mathematics, vol. 1, no. 4 pp. 30-40, 2008.

5. F. Belzunce, J. Navarro, J. M. Ruiz, and Y. Aguila, Some results on residual entropy function, Metrika, vol. 59, no. 2, pp. 147-161, 2004.

6. A. Di Crescenzo, and M. Longobardi, Entropy-based measure of uncertainty in past lifetime distributions, Journal of Applied Probability, vol. 39, no. 2, pp. 434-440, 2002.

7. A. Di Crescenzo, and M. Longobardi, A measure of discrimination between past lifetime distributions, Statistics and Probability Letters, vol. 67, no. 2, pp. 173-182, 2004

8. N. Ebrahimi, How to measure uncertainty about residual lifetime, Sankhya A, vol. 58, no. 1, pp. 48-57, 1996.

9. N. Ebrahimi, and F. Pellerey, New partial ordering of survival functions based on notation of uncertainty, Journal of Applied Probability, vol. 32, pp. 202-211, 1995.

10. J. Galambos, and S. Kotz, Characterization of probability distributions: a unified approach with an emphasis on exponential and related models, Lecture notes on Mathematics 675, Springer-Verlag, Berlin, 1978.

11. V. Kumar, H. C. Taneja, and R. Srivastava, A dynamic measure of inaccuracy between two past lifetime distribution, Metrika, vol.74, no. 1, pp. 1-10, 2011.

12. F. Misagh, and G.H. Yari, On weighted interval entropy, Statistics and Probability Letters, vol. 81, no. 2, pp. 188-194, 2011.

13. F. Misagh, and G.H. Yari, Interval entropy and informative distance, Entropy, vol. 14, pp. 480-490, 2012.

14. K. R. M. Nair, and G. Rajesh, Characterization of probability distributions using the residual entropy function, Journal of the Indian Statistical Association, vol. 36, pp. 157-166, 1998.

15. A. K. Nanda, and P. Paul, Some properties of past entropy and their applications, Metrika, vol. 64, no. 1, pp. 47-61, 2006.

16. J. Navarro, and J. M. Ruiz, Faliure rate function for doubly truncated random variables., IEEE Transactions on Information Theory, vol. 45, pp. 685-690, 1996. 
17. J. Navarro, and J. M. Ruiz, Characterization from relationships between failure rate functions and conditional moments communications in statistics: theory methods, vol. 33, no. 12, pp. 3159-3171, 2006.

18. M. Shaked, and J. G. Shanthikumar, Stochastic Orders and Their Applications, Academic Press, New York, 1994.

19. C. E. Shannon, A mathematical theory of communication, Bell System Technical Journal, vol. 27, 379-432, 1948.

20. S.M. Sunoj, P. G. Sankaran, S. S. Maya, Characterization of life distribution using conditional exceptations of doubly (Interval) truncated random variables, Communications in Statistics: Theory and Methods, vol. 38, no. 9, pp.1441-1452, 2009.

21. R. S. Verma, Generalization of Renyi's entropy of order $\alpha$, Journal of Mathematical Sciences, vol. 1, pp. 34-48, 1966.

22. S. Treanta Optimization on the distribution of population densities and the arrangement of urban activities, Statistics, Optimization and Information Computing, vol.6, pp. 208-218, 2018.

23. C. Kundu, On weighted measure of inaccuracy for doubly truncated random variables, Communication in Statistics: Theory and Methods, vol.46, pp. 3135-3147, 2017.

24. S. Kayal, and R. Moharana, Some Results on a doubly truncated generalized discrimination measure. 46, 3135-3147., Applications of mathematics, vol.5, pp. 585-605, 2016. 